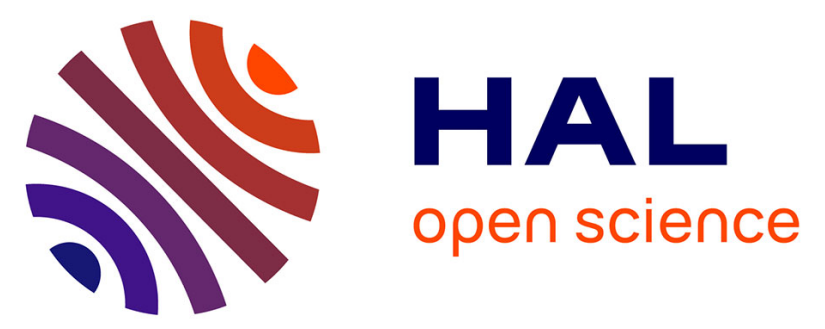

\title{
Development of a Novel Experimental Device to Investigate Swelling of Elastomers in Biodiesel Undergoing Multiaxial Large Deformation
}

Shiau Ying Ch'ng, Andri Andriyana, Erwan Verron, Omid Kahbasi, Roslina Ahmad

\section{To cite this version:}

Shiau Ying Ch'ng, Andri Andriyana, Erwan Verron, Omid Kahbasi, Roslina Ahmad. Development of a Novel Experimental Device to Investigate Swelling of Elastomers in Biodiesel Undergoing Multiaxial Large Deformation. Experimental Mechanics, 2013, 53 (8), pp.1323-1332. 10.1007/s11340-013-9737-

2. hal-03284768

\section{HAL Id: hal-03284768 https://hal.science/hal-03284768}

Submitted on 18 Aug 2021

HAL is a multi-disciplinary open access archive for the deposit and dissemination of scientific research documents, whether they are published or not. The documents may come from teaching and research institutions in France or abroad, or from public or private research centers.
L'archive ouverte pluridisciplinaire HAL, est destinée au dépôt et à la diffusion de documents scientifiques de niveau recherche, publiés ou non, émanant des établissements d'enseignement et de recherche français ou étrangers, des laboratoires publics ou privés. 


\title{
Development of a Novel Experimental Device to Investigate Swelling of Elastomers in Biodiesel Undergoing Multiaxial Large Deformation
}

\author{
S.Y. Ch'ng • A. Andriyana • E. Verron • O. Kahbasi • \\ R. Ahmad
}

\begin{abstract}
The lifetime of an elastomeric product depends on the nature of mechanical loading and the environmental condition during the service. In this context, at least two important aspects contribute to the degradation of the elastomeric parts in service: diffusion of aggressive liquids leading to swelling and fluctuating multiaxial mechanical loading leading to fatigue failure. Moreover, the amount of swelling of elastomers in solvent is affected by the presence of mechanical loading. Hence, it is essential to understand the interactions between the two phenomena for durability analysis of the component. The present study investigates the swelling of elastomers due to diffusion of palm biodiesel in the presence of static multiaxial large deformation. For this purpose, new experimental device and specimen are developed. The device consists of a hollow diabolo elastomeric specimen attached to specially-designed circular metallic grips and plates such that immersion tests can be conducted while the specimens are simultaneously subjected to various mechanical loadings: simple tension, simple torsion and combined tension-torsion. Thus, diffusion of liquids takes place in the material which concurrently undergoes multiaxial large deformation. Two types of elastomers are investigated: Nitrile Rubber (NBR) and Polychloroprene Rubber (CR). The particular features of the device and specimen are discussed and perspectives for further improvement are drawn.
\end{abstract}

S.Y. Ch'ng · A. Andriyana $(\bowtie) \cdot$ O. Kahbasi · R. Ahmad Department of Mechanical Engineering, University of Malaya, 50603 Kuala Lumpur, Malaysia

e-mail: andri.andriyana@um.edu.my

E. Verron

Institut de Recherche en Génie Civil et Mécanique (GeM), LUNAM Université, UMR CNRS 6183, Ecole Centrale de Nantes, BP 92101, 44321 Nantes cedex 3, France
Keywords Elastomer · Palm biodiesel · Swelling · Multiaxial large strain $\cdot$ Hydrostatic stress

\section{Introduction}

Elastomers have been a popular and desirable choice for numerous applications in industry mainly due to their interesting mechanical characteristics [1]. Typical industrial usages of elastomers include flexible pipes, tires, bearing, seals, o-rings, gasket, lining, etc. During their service, the elastomeric components are often subjected to fluctuating mechanical loading which could lead to fatigue failure [2]. Furthermore, in many sealing systems such as o-ring or gaskets, they are exposed to aggressive liquids or solvents in addition to multiaxial large deformations [3]. The solvent can penetrate to the stressedelastomeric components which could lead to the degradation of materials in the form of swelling. Indeed, the presence of solvent in the rubber matrix weakens the matrix and decreases its resistance to failure [4]. It is to note that the resulting equilibrium swelling level depends on the elastomer-solvent combination and on the presence of mechanical loading [5]. Hence, the understanding and consideration of interactions between the two phenomena, i.e. degradation due to fluctuating mechanical loading and degradation due to swelling in the presence of mechanical loading, is an essential pre-requisite for the durability analysis of such components. In the present paper, only degradation in elastomers due to swelling in the presence of static multiaxial mechanical loading is addressed.

Swelling of elastomers had been extensively studied by Flory [6] and Treloar [7] who investigated the swelling of stress-free rubber in solvents. Following these works, a 
number of studies are available in the literature dealing with the diffusion of liquids in elastomers undergoing uniaxial loading [8-16]. In the case of uniaxially stressed rubber, it was found that the tensile stress increases the liquid uptake while opposite phenomenon is observed in the presence of compressive stress [9, 17, 18]. Treloar [7] attributed these phenomena to the hydrostatic part of the Cauchy stress in the materials. The author provided the same argument for the decrease in swelling of rubber solid cylinder undergoing simple torsion. According to the author, the introduction of simple torsion generates the compressive stress along radial direction of the cylinder and thus limits the liquid uptakes. Similar results were obtained by Loke et al. [19]. It is important to highlight that in these works, the authors considered the swelling of solid hyperelastic cylinder in solvent undergoing simple torsion with small twists, i.e. only small strain problems were studied. The extensions to the large strain simple torsion or general multiaxial loading was not provided. Thus, the effect of the presence of multiaxial mechanical loading on the level of liquid uptake in elastomers is not established.

The objective of the present work is to investigate the swelling of elastomers in biodiesel in the presence of static multiaxial large strain. For this purpose, novel experimental specimen and device are developed. The proposed experimental setup enables immersion tests to be conducted while the specimens are simultaneously subjected to various mechanical loadings: simple tension, simple torsion and combined tension-torsion. Hence, diffusion of liquid into rubber occurs in the presence of multiaxial strain state. While palm biodiesel is used in the present study, it is to note that the experimental setup can be used for various combinations of elastomer-solvent without major difficulty. The details of experimental device and specimen are provided in Section "Experimental Program". Results are presented in Section "Results" and discussions are presented in Section "Discussion" while concluding remarks are given in Section "Conclusions".

\section{Experimental Program}

\section{Materials}

Specially designed elastomers are purchased from MAKA Engineering Sdn. Bhd., Malaysia. The as-received elastomeric specimens investigated in this paper are commercial grades of NBR and CR with 60 shore hardness and $25 \%$ of carbon black content. Palm biodiesel is provided by Mission Biotechnologies Sdn. Bhd. All immersion tests are conducted in $100 \%$ palm biodiesel at room temperature. Due to confidentiality constraint, detailed recipes of rubbers and biodiesel are not provided.
Experimental Device and Specimen

In order to investigate the diffusion of palm biodiesel into elastomers undergoing multiaxial large strain, speciallydesigned specimen and device are developed. They consist of a hollow diabolo elastomeric specimen equipped with circular metallic grips and plates as shown in Figs. 1 and 2. The main features of the device and specimen are summarized as follow:

1. The device consists of four identical metallic grips which are screwed together at both ends of the elastomeric specimen. The inner part of the metallic grips is designed to fit in the hexagonal shape of the specimen. In this way, twist and axial extension/contraction can be imposed simultaneously to the specimen while the immersion test is carried out.

2. Each metallic grip consists of six identical holes which are symmetrically arranged along circumferential direction. Different twist can be applied to the specimen by twisting the upper part of metallic grip while fixing the lower part of the grip. Once the desired angle is obtained, bolts are inserted through the holes located at the lower and upper parts of the grip in order to maintain constant twist. Axial extension or contraction can be imposed by adjusting vertically the position of the nuts in the bolts as illustrated in Fig. 3.

3. The rubber specimen has a diabolo-like shape with a hollow inside to allow the biodiesel to diffuse into the specimen from its inner and outer wall surfaces. Thus, it is expected that equilibrium swelling can be achieved within a reasonable period of time.

4. After the metallic grips are tightly screwed to the specimen, thin metallic plates are attached at both ends in order to prevent the diffusion of liquids from both specimen ends. Two o-rings with different diameter are inserted between plates and grips to provide a tight seal.

Immersion Tests and Swelling Measurement

As mentioned previously, our particular device and specimen allow the immersion test to be conducted on the specimens while they are subjected to different mechanical loading conditions. In the present study, 16 different mechanical loading conditions are investigated. The details of these conditions are given in Table 1 .

An illustration of the specimen and device before and after the application of mechanical loading, i.e. tension and torsion, is provided in Fig. 3.

After the desired axial extension $(\Delta L)$ and twist angle $(\theta)$ have been applied, the specimens are completely immersed in palm biodiesel for 1 week $(170 \mathrm{~h})$ at room temperature. In order to determine the swelling level, the mass 
Fig. 1 Exploded view of the experimental device and specimen

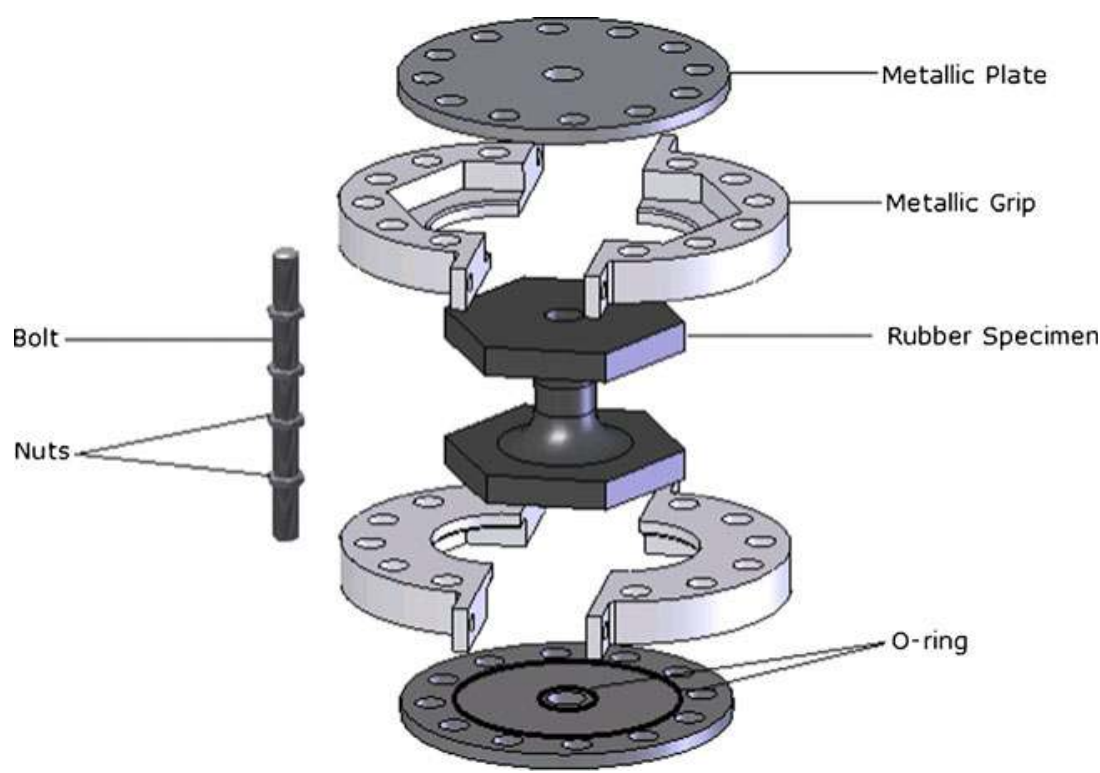

of the specimens before and after immersion test is measured. The detailed procedures of immersion test can be summarized as follow:

1. Before immersion test, the mass of the dry rubber specimen is measured in air and in distilled water using digital weighting machine.

2. The rubber specimen is subsequently attached into the experimental device as described in the previous section in order to impose the mechanical loading.

3. The experimental devices are then immersed into palm biodiesel for 1 week at room temperature.

4. After 1 week, the devices are removed from the biodieseland the rubber specimens are dismantled from the metallic plates and grips. The specimens are then quickly dipped into acetone and cleaned with filter paper to remove excessive oil.

5. The mass of the specimens after immersion is measured as in step 1 . The percentage of mass change and volume change are calculated using the following simple relation [20]:

$\%$ Mass Change $=\frac{M_{2}-M_{1}}{M_{1}} \times 100$

$\%$ Volume Change $=\frac{\left(M_{2}-M_{4}\right)-\left(M_{1}-M_{3}\right)}{\left(M_{1}-M_{3}\right)} \times 100$

where $\mathrm{M}_{1}$ and $\mathrm{M}_{2}$ are the masses in air (gram) before and after immersion while $\mathrm{M}_{3}$ and $\mathrm{M}_{4}$ are the masses in water (gram) before and after immersion.

\section{Results}

Figure 4 shows the percentage of mass change and volume change of NBR immersed during 1 week in palm biodiesel
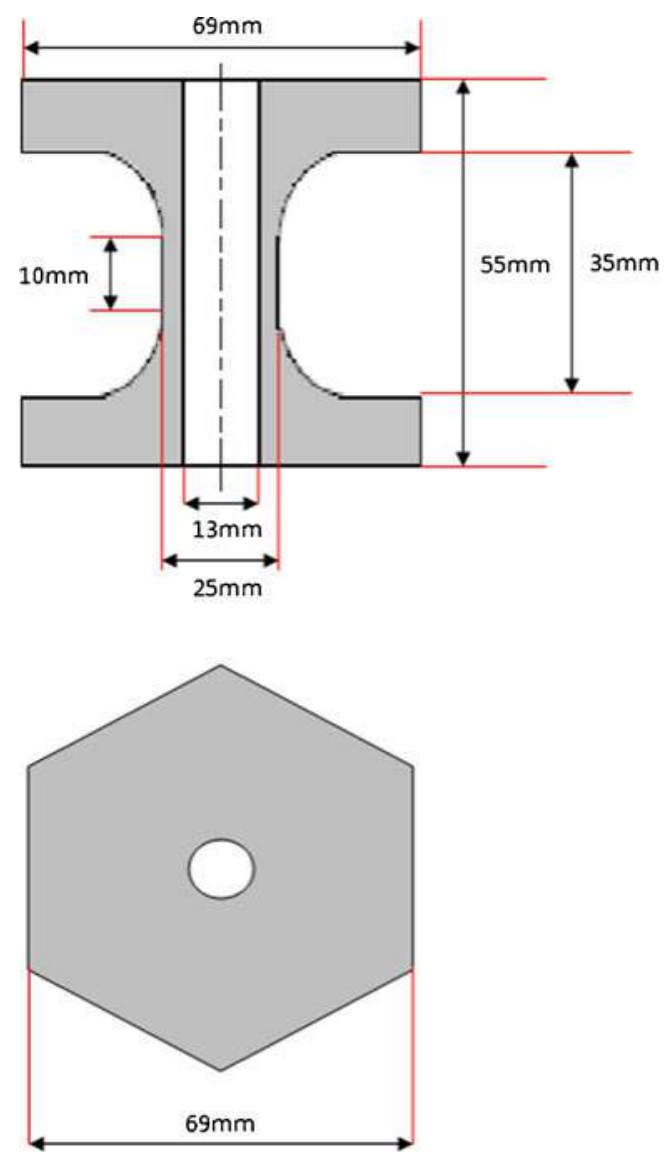

Fig. 2 Geometry of rubber specimen 
Table 1 Mechanical loading conditions

\begin{tabular}{|c|c|c|c|c|}
\hline \multirow[t]{3}{*}{ Specimen } & \multicolumn{4}{|l|}{ Applied load } \\
\hline & \multicolumn{2}{|l|}{ Tensile load } & \multicolumn{2}{|c|}{ Torsional load } \\
\hline & $\begin{array}{l}\text { Axial extension } \\
\Delta L(\mathrm{~mm})\end{array}$ & $\begin{array}{l}\text { Resulting stretch ratio } \\
\lambda=L / L_{o}\end{array}$ & $\begin{array}{l}\text { Twist angle } \\
\theta\left(^{\circ}\right)\end{array}$ & $\begin{array}{l}\text { Resulting twist per unit length } \\
\gamma(\mathrm{rad} / \mathrm{mm})\end{array}$ \\
\hline S0T0 & 0 & 1 & 0 & 0 \\
\hline S0T30 & 0 & 1 & 30 & 0.021 \\
\hline S0T60 & 0 & 1 & 60 & 0.042 \\
\hline S0T90 & 0 & 1 & 90 & 0.063 \\
\hline S20T0 & 5 & 1.2 & 0 & 0 \\
\hline S20T30 & 5 & 1.2 & 30 & 0.017 \\
\hline S20T60 & 5 & 1.2 & 60 & 0.035 \\
\hline S20T90 & 5 & 1.2 & 90 & 0.052 \\
\hline S40T0 & 10 & 1.4 & 0 & 0 \\
\hline S40T30 & 10 & 1.4 & 30 & 0.015 \\
\hline S40T60 & 10 & 1.4 & 60 & 0.029 \\
\hline S40T90 & 10 & 1.4 & 90 & 0.045 \\
\hline S60T0 & 15 & 1.6 & 0 & 0 \\
\hline S60T30 & 15 & 1.6 & 30 & 0.013 \\
\hline S60T60 & 15 & 1.6 & 60 & 0.026 \\
\hline S60T90 & 15 & 1.6 & 90 & 0.039 \\
\hline
\end{tabular}

under different loading conditions: initially stress-free S0T0, simple tension S20T0, S40T0, S60T0, simple torsion S0T30, S0T60, S0T90, and combined tension-torsion S20T30, S20T60, S20T90, S40T30, S40T60, S40T90, S60T30, S60T60, S60T90. For each condition, three specimens were tested. In the absence of tensile strain, the percentage of mass changes varies from 1.44 to $1.65 \%$, while the percentage of volume changes varies from 2.41 to $2.62 \%$. The effect of mechanical loading, in particularly tensile strain, on the amount of swelling is clearly shown in this figure. In the presence of $60 \%$ tensile strains, the swelling ranges from 2.25 to $2.93 \%$ (on mass basis) and from 3.60 to $4.55 \%$ (on volume basis). In contrast, the effect of twist on the swelling level appears to be not as significant as the effect of tensile strain. Indeed, the application of twist increases only slightly the amount of liquid uptakes. The effect of torsion on the amount of swelling is discussed in detail in Section "Mechanical Quantity Affecting Swelling Level"

Similar immersion tests are conducted on CR specimens and the corresponding results are presented in Fig. 5. It is found that for a given mechanical loading, the swelling level in $\mathrm{CR}$ is consistently higher than the one in NBR. In the absence of tensile strain, the swelling in CR ranges from 4.7 to $5.82 \%$ (on mass basis) and from 10.14 to $11.32 \%$ (on volume basis). In the presence of $60 \%$ tensile strains, it ranges from 11.16 to $12.29 \%$ (on mass basis) and from 19.12 to $21.34 \%$ (on volume basis). Similarly to NBR,
Fig. 3 Experimental device: (a) with no mechanical loading (initially stress-free) and (b) under twist angle $\theta$ and axial extension $\Delta L$. For practical purposes, the initial distance between two metallic plates is chosen as the initial length $L_{o}$ during the experiment

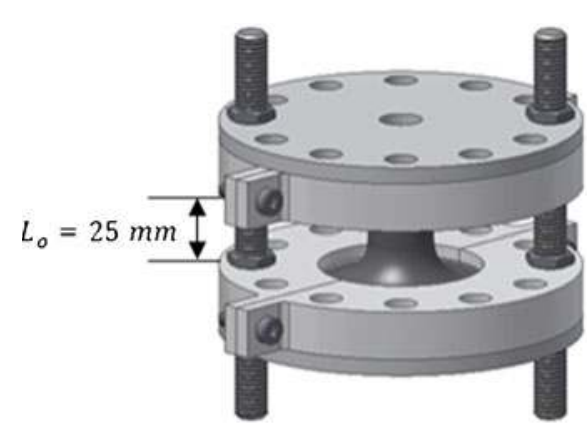

(a)

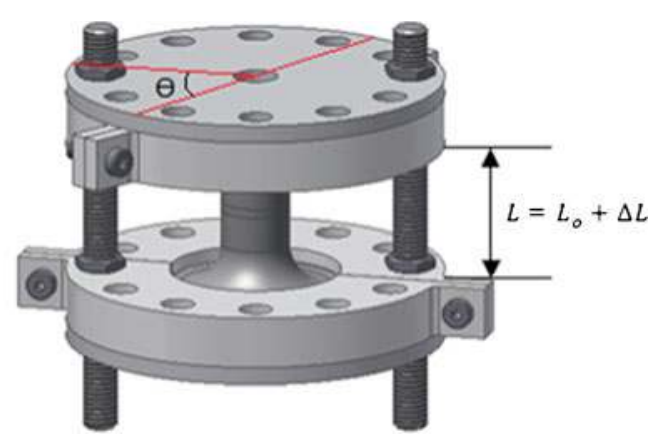

(b) 
Fig. 4 (a) Mass change and (b) volume change of NBR after 1 week immersion in palm biodiesel (a)

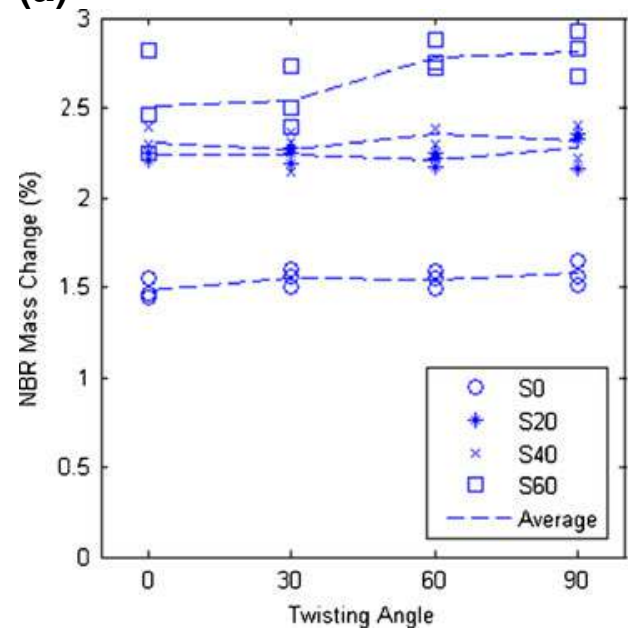

(b)

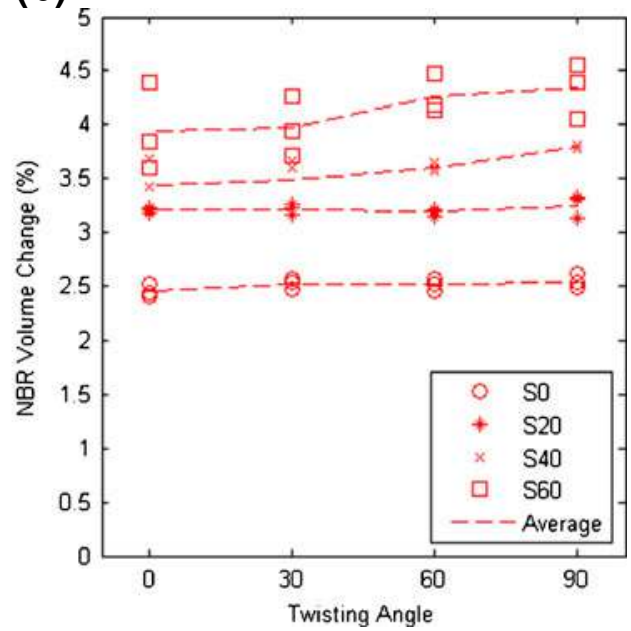

the tensile strain appears to have more significant effect than torsion on swelling level.

The significant increase of swelling due to the application of tensile strain can be explained by the fact that the initial available surface of the specimen through which diffusion occurs increases [9]. Moreover, tensile strain generates tensile stress in the material. In this case, the hydrostatic part of the stress is negative. According to Treloar [7], negative hydrostatic part of the stress facilitates the liquid uptake.

From a molecular standpoint, CR is made from emulsion polymerization of 2-chloro-1, 3-butadiene while NBR is made from emulsion copolymer of acrylonitrile and butadiene. The polar substituent of acrylonitrile in NBR and chlorine substituent in $\mathrm{CR}$ contribute to their resistance to mineral oils [21]. However, the tendency of rubber to swell in solvent generally follows the principle of like dissolve like: polar solvent are more likely to dissolve polar substances and non-polar substances are more likely to dissolve in non-polar solvent [22]. The high polarity of ester in palm biodiesel favors the forming of polymer-solvent interaction in CR and high polarity NBR [23]. Therefore, for engineering applications involving the contamination of highly polar solvent such as palm biodiesel in highly polar elastomeric components (e.g. CR and high polarity NBR), caution should be taken so that the resulting swelling does not significantly decrease the performance of the component during the service.

Remark 1 Treloar [5] conducted the swelling tests on Natural Rubber (NR) in benzene and heptane for tensile strains which range between 50 and $150 \%$ until the specimens reach equilibrium swelling. While the resulting swelling level in the author's work cannot be directly compared to our results due to different rubber-solvent combination and different immersion duration, both tests lead to the same qualitative conclusion: tensile strain increases swelling.
Fig. 5 (a) Mass change and (b) volume change of $\mathrm{CR}$ after 1 week immersion in palm biodiesel (a)

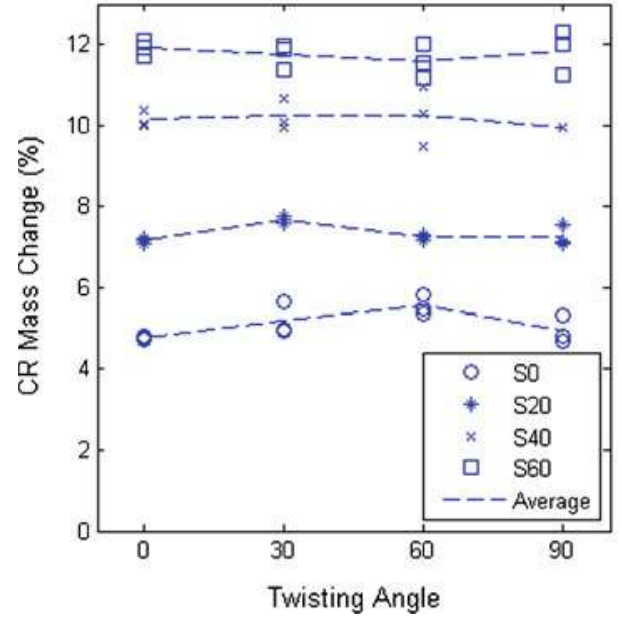

(b)

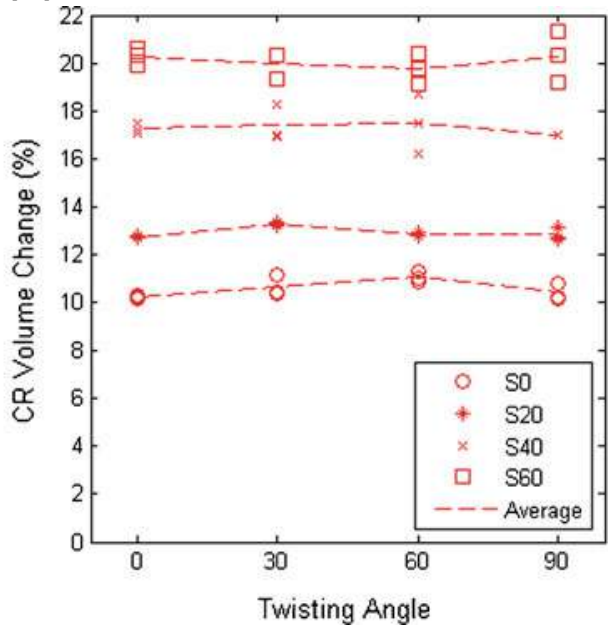


Remark 2 Loke et al. [19] and Treloar [24] conducted the swelling tests on Natural Rubber (NR) and Synthetic Natural Rubber (IR) in toluene for twist per unit length levels which range between 0 and $0.05 \mathrm{rad} / \mathrm{mm}$ until the specimens reach equilibrium swelling. The above twist is comparable to the one conducted in the present work ( 0 to $0.063 \mathrm{rad} / \mathrm{mm}$ ). However, the resulting swelling levels in the two works cannot be directly compared due to different rubber-solvent combination and different immersion duration. Moreover, the two tests do not lead to the same conclusion with regard to the effect of torsion. For Loke et al. [19] and Treloar [24], torsion decreases swelling. In the present work, torsion increases slightly swelling. As detailed in the Section "Mechanical Quantity Affecting Swelling Level", this discrepancy can be related to the geometry of the specimen used during the test.

\section{Discussion}

Discussion on the Experimental Device and Specimen

Before proceeding further, it is important to highlight the advantages and limitations of the device and specimen for further improvement. Indeed, the main advantages are summarized below:

1. Multiaxial strain can be introduced simultaneously to the specimens during the immersion test. This will allow the observation on the effect of mechanical loading on the diffusion of liquids.

2. Diffusion of liquids practically occurs only along radial direction, i.e. simple one dimension diffusion. Thus, the corresponding experimental results can be used to validate simple mechanical models for investigating the swelling of elastomers in solvent undergoing simultaneously mechanical loading.

3. Hollow diabolo specimen ensures that the equilibrium swelling can be achieved within a reasonably short period of time while permitting the application of moderate compressive strain without buckling or wrinkling.

4. Different twist angles and axial extensions/contractions can be imposed to the specimen simply by fixing and adjusting bolts and nuts at appropriate positions.

5. No adhesive is used in the connection between the metallic part and the rubber specimen. Thus, immersion tests can be conducted using any types of aggressive liquids since debonding between rubber and metallic parts is not an issue.

6. The metallic grips and plates can be easily attached and dismantled from the rubber specimen.
7. The metallic grips are designed so that the device and specimen can be used for several subsequent mechanical testing which follow the immersion tests such as uniaxial tensile, simple torsion, combined torsiontension or fatigue testing.

Although the device enables the experiment set up to be carried out successfully, there are some limitations that need to be improved in future works:

1. Under simple torsion, the specimen is subjected to nonuniform strain (or stress) field. Being preliminary work, this additional difficulty dealing with diffusion of liquids in the presence of non-uniform strain (or stress) should be avoided. A relatively uniform strain and stress fields in the specimen under torsion can be obtained by sufficiently increasing specimen inner diameter while fixing its wall thickness.

2. Different parts in the specimen undergo different swelling levels due to specimen geometry. It is preferable to confine the diffusion, i.e. swelling, in the middle part of the specimen since this is the focus area of our study. This can be obtained by introducing appropriate sealing on the specimen such that diffusion takes place only through the inner and outer surfaces of the middle part of the specimen.

Once the swelling localizes at the middle part, the amount of swelling can be calculated by considering the change in volume or mass of the middle part only, and not the whole specimen. Works are currently in progress in order to overcome the above limitations and difficulties.

\section{Mechanical Quantity Affecting Swelling Level}

As mentioned in the previous section, the reduction or increase in the swelling is mainly governed by the hydrostatic part of the Cauchy stress [7]. A tensile stress (negative hydrostatic stress) favors swelling while a compressive stress (positive hydrostatic stress) restricts swelling. In the present study, complex static mechanical loading conditions such as combined tension-torsion are addressed. Hence, the calculation of the hydrostatic part of the Cauchy stress in the specimen requires the analytical solution of hyperelastic hollow cylinder under corresponding mechanical loading conditions: simple tension, simple torsion and combined torsion-tension.

During the immersion tests, the elastomeric specimens are subjected to constant axial extension and constant twist. Due to viscoelastic behavior of the materials, the resulting stress decreases with time during the immersion, i.e. the materials experience stress relaxation. The viscoelastic characteristic of our materials is highlighted in Fig. 6. The decrease of stress due to viscoelasticity yields also to the 
decrease of the hydrostatic part of stress. Since the precise kinetic of stress-relaxation during the immersion test is not known, i.e. the stress level in the material at any particular time is not known, we only focus on the initial hydrostatic stress in the dry elastomers before the immersion test.

In Fig. 6, the engineering stress-engineering strain responses of dry NBR and dry CR under cyclic loading conditions are presented. The tests were conducted at room temperature and at a displacement rate of $0.5 \mathrm{~mm} / \mathrm{s}$. It is observed that both materials exhibit strong inelastic responses: stress-softening (Mullins effect), mechanical hysteresis (viscoelasticity) and permanent set. After four cycles of loading, stress-softening disappears while the hysteresis stabilizes. For the sake of simplicity, in the present study, the analytical determination of the hydrostatic stress is only based on the first uploading response of the materials, i.e. the viscoelastic response and stress-softening are neglected. Moreover, both dry NBR and dry CR are assumed to be isotropic and incompressible which obeys neo-Hookean hyperelastic constitutive equation.

The complete derivation of the governing equations for an isotropic incompressible hyperelastic tube under combined tension-torsion can be found for example in Green and Adkins [25], and with the present notations in Adriyana et al. [26] and Vernon and Adriyana [27]. Using cylindrical coordinates system, for neo-Hookean constitutive equation, the resulting Cauchy stress tensor at any particular point of the undeformed configuration is given by:

$$
\begin{aligned}
\sigma(R)= & C \lambda \gamma^{2}\left(R^{2}-R_{o}^{2}\right) e_{r} \otimes e_{r}+C \lambda \gamma^{2}\left(3 R^{2}-R_{o}^{2}\right) \\
& \times e_{\theta} \otimes e_{\theta}+2 C \lambda^{3 / 2} \gamma R\left(e_{\theta} \otimes e_{z}+e_{z} \otimes e_{\theta}\right) \\
& +\left[2 C\left(\lambda^{2}-\frac{1}{\lambda}\right)+C \lambda \gamma^{2}\left(R^{2}-R_{o}^{2}\right)\right] e_{z} \otimes e_{z}
\end{aligned}
$$

where $\left(e_{r}, e_{\theta}, e_{z}\right)$ represent the three unit vectors along radial, circumferential and axial directions respectively in the deformed configuration. $\lambda$ is the stretch ratio and $\gamma$ is the twist per unit length. For each loading condition, their values are tabulated in Table $1 . R$ is the radial position of any particular point in the undeformed configuration while $R_{O}$ is the initial outer radius of the cylinder. $C$ is the material parameter, identified from the first uploading stress response as mentioned in the previous paragraph. The hydrostatic stress is obtained by taking the trace of the Cauchy stress tensor [28]:

$p=-\frac{1}{3} \operatorname{tr}(\sigma)$

which yields to:

$$
\begin{aligned}
p(R)=-\frac{1}{3}[ & C \lambda \gamma^{2}\left(R^{2}-R_{o}^{2}\right)+C \lambda \gamma^{2}\left(3 R^{2}-R_{o}^{2}\right) \\
& \left.+2 C\left(\lambda^{2}-\frac{1}{\lambda}\right)+C \lambda \gamma^{2}\left(R^{2}-R_{o}^{2}\right)\right]
\end{aligned}
$$

For each loading condition, the initial hydrostatic stress in the middle part of the specimen at various radial positions $R$ is calculated and depicted in Fig. 7 for both NBR and CR. In this calculation, the values of $C$ for dry NBR and dry CR are $0.554 \mathrm{MPa}$ and $0.594 \mathrm{MPa}$ respectively. Moreover, the inner and outer radii are $R_{i}=6.5 \mathrm{~mm}$ and $R_{O}=12.5 \mathrm{~mm}$ respectively. For a given twist, it is observed that the hydrostatic stress decreases with the increase of radial position. More precisely, under torsion the elastomeric cylinder experiences compressive stress at the inner surface and tensile stress at the outer surface, i.e. the strain is non-uniform. Thus, the swelling occurs in the presence of non-uniform strain (stress) field. As shown in Fig. 7, there exists a particular plane along which the hydrostatic stress is zero. This particular plane is located at the critical radius $R_{c}=\sqrt{0.6} \cdot R_{O}$ which is independent of the applied twist. Between the inner surface and the particular plane, i.e. $R_{i}<R<R_{c}$, the hydrostatic stress is positive (in compression state) while between the particular plane and the outer surface of the cylinder, i.e. $R>R_{c}$, the hydrostatic stress is negative (in tension state). When the twist increases, the hydrostatic stress gradient also increases, i.e. increasing the non-uniformity of strain (stress) field in the materials. Furthermore, it appears that the effect of tensile loading is to translate vertically downward the curve of hydrostatic stress, i.e. to a more negative value. As indicated in
Fig. 6 Stress-strain curves of (a) NBR and (b) CR at dry states under cyclic loading condition (a)

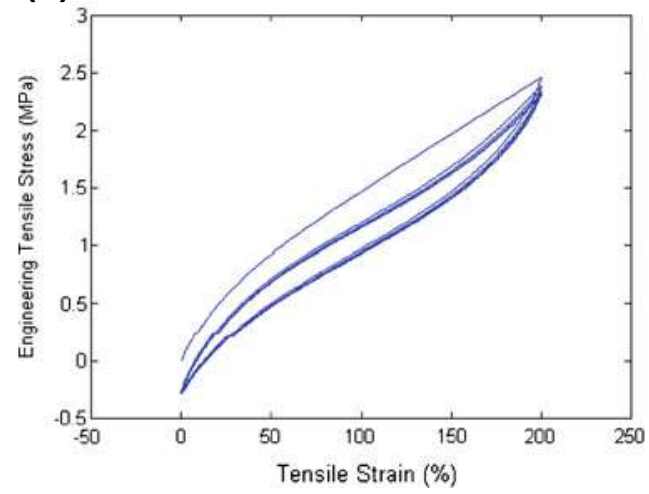

(b)

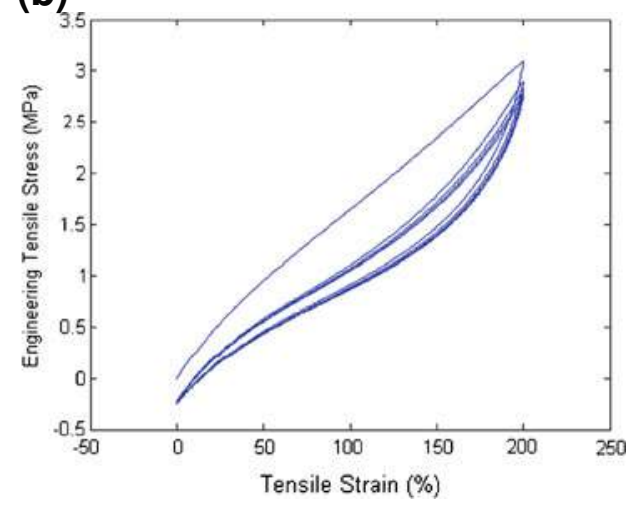


Fig. 7 Initial hydrostatic part of Cauchy stress at different radial position for (a) NBR and (b) CR (a)

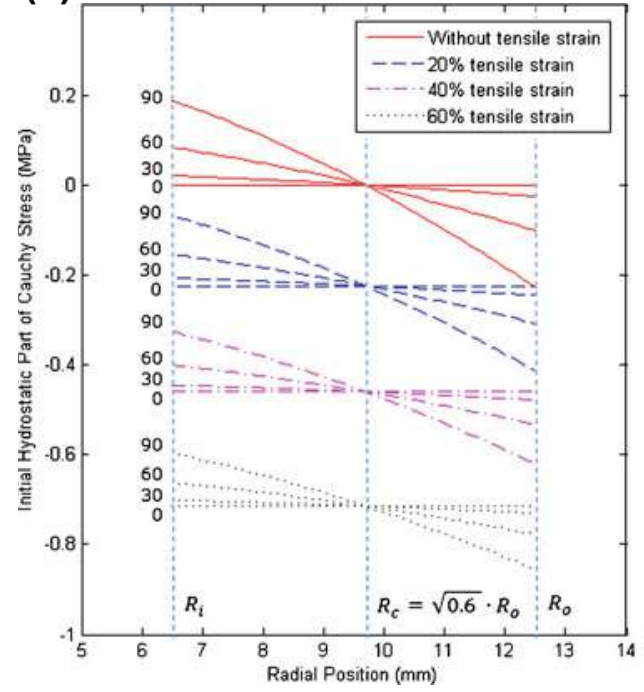

(b)

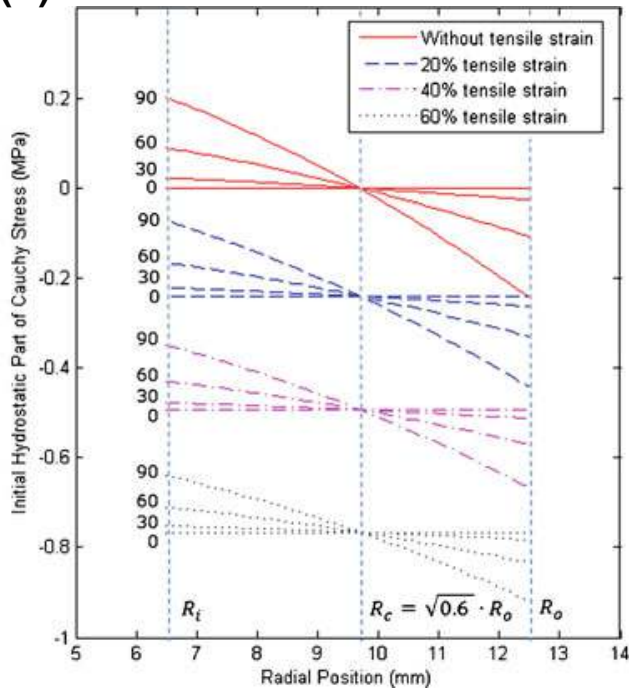

Fig. 7, the amount of this translation decreases slightly with the radial position. Finally, as the tensile loading increases, its effect becomes more significant which yields to the reduction of the non-uniformity of strain (stress).

Since the strain and stress are non-uniform in the rubber specimen, the average value of hydrostatic stress has to be calculated. Figure 8 presents the average hydrostatic stress for different loading conditions. In this case, the average hydrostatic stress is calculated from:

$\bar{p}=\frac{1}{R_{o}-R_{i}} \int_{R_{i}}^{R_{o}} p \cdot d r$

As shown in Fig. 8(a) and (b), the tensile strain affects significantly the average value of hydrostatic stress. In contrast, the average value of hydrostatic stress does not change significantly with the increase of twist. Indeed, for the case of simple torsion, the average hydrostatic stresses in both NBR and CR are close to zero regardless the amount of twist. For the sake of clarity, the corresponding average hydrostatic stress is re-plotted in Fig. 8(c) and (d). These results can be helpful in understanding why the swelling levels in our materials are not greatly affected by the amount of twist.

To gain an insight on the effect of simple torsion on the swelling level for our specimen, the percentage of mass change is plotted against the average hydrostatic stress for simple torsion cases in Fig. 9. When twist is applied, it is found that the resulting swelling level is systematically higher than the swelling in initially stress-free specimens. The increase of swelling due to the application of twist is in contrast to the prediction of Loke et al. [19] and Treloar [24]. In our opinion, the corresponding discrepancy could be attributed to the geometry of the specimen. Indeed, while hollow cylindrical specimen is considered in the present study, Loke et al. [19] and Treloar [24] used a solid cylindrical specimen. For a given twist, the zone of the cylinder experiencing compressive hydrostatic stress will be larger in the case of solid cylinder than that in the case of hollow cylinder. As discussed and detailed in the previous paragraph, the zone with compressive hydrostatic stress is located at $R_{i}<R<R_{c}$. Note that in the case of solid cylinder, $R_{i}=0$. Since the zone experiencing compressive hydrostatic stress in solid cylinder is larger, the resulting average hydrostatic stress becomes higher, i.e. more positive, higher average compressive hydrostatic stress. Thus, under a given twist, the resulting swelling in solid cylinder is lower than that in hollow cylinder.

While the introduction of torsion appears to increase slightly swelling, our results do not clearly indicate the effect of the amount of torsion on the swelling level. The corresponding observation could be attributed to the particular geometry of our specimen. As illustrated in Fig. 8, the change in the average hydrostatic pressure due to the increase in torsion is not as significant as due to the application of tensile strain.

Finally, the above observations suggest that the effect of torsion on swelling is dictated by the specimen geometry. In the case of solid cylinder, the resulting average hydrostatic stress is positive, i.e. swelling is restricted. Thus, lower swelling is expected to occur than the one in stressfree cylinder. This case corresponds to the works of Loke et al. [19] and Treloar [24]. However, when a hollow cylinder having inner radius larger than the critical radius is used, i.e. with $R_{i}>R_{c}$, the resulting average hydrostatic stress is negative and significantly higher swelling is expected to occur than the one in stress-free cylinder. It is straightforward to show that the condition $R_{i}>R_{c}$, is equivalent with the ratio $\frac{t}{R_{m}}<0.259$, where $t=R_{o}-R_{i}$ 
Fig. 8 Average initial hydrostatic part of Cauchy stress at different twisting angle for (a) NBR, (b) CR, (c) NBR without tensile strain and (d) $\mathrm{CR}$ without tensile strain (a)

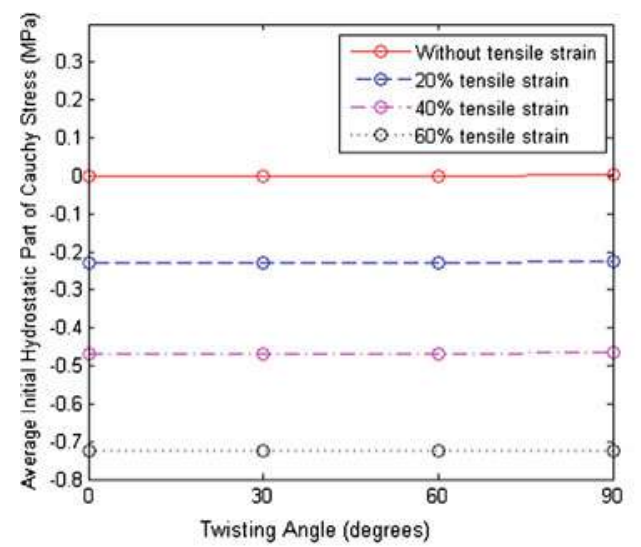

(c)

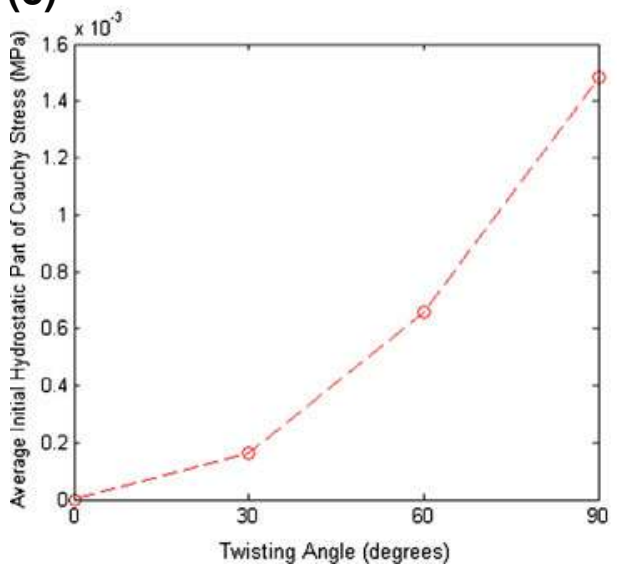

(b)

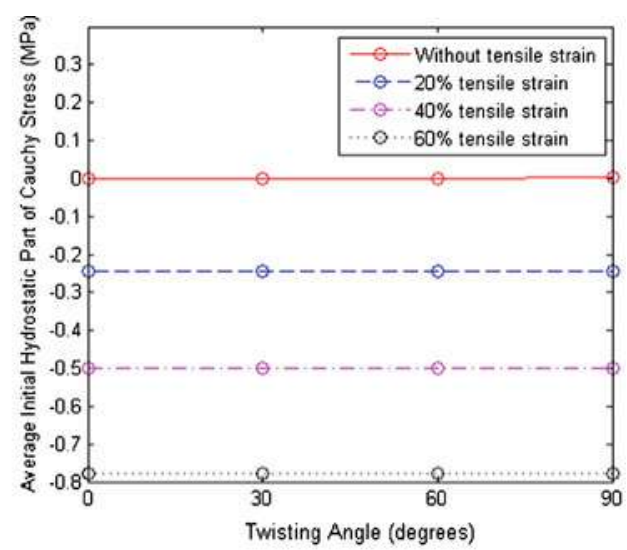

(d)

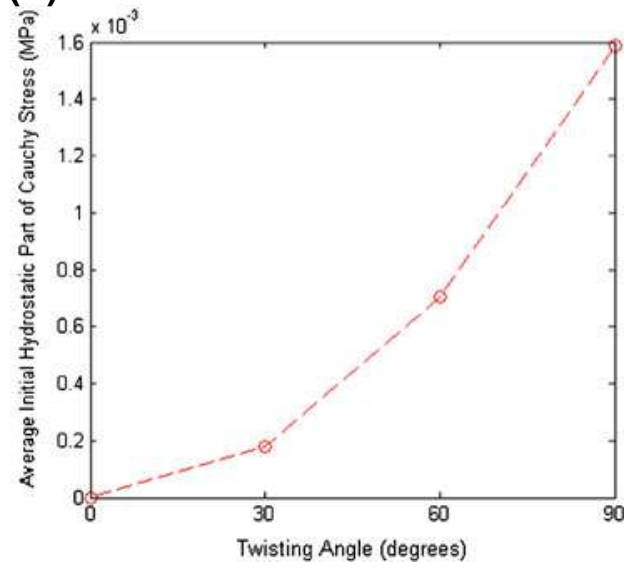

is the wall thickness and $R_{m}=\frac{1}{2}\left(R_{o}+R_{i}\right)$ is the mean radius of the cylinder. For our purpose, thin-walled cylinder with the above ratio would be preferable than the solid one since certain level of swelling can be reached within a relatively shorter period of time. Moreover, the resulting stress and strain fields will be relatively more uniform. The middle part of the elastomeric specimens used in the present study has the thickness-mean radius ratio of $\frac{t}{R_{m}}=$ 0.632 resulting to difficulty in obtaining clear influence of torsion on the swelling level. Thus, further works and validations using improved specimens having the above ratio, i.e. with $\frac{t}{R_{m}}<0.259$, are required in order to fully establish the effect of static multiaxial loading on the swelling of elastomers.
Fig. 9 Mass change as a function of average hydrostatic component of stress for (a) NBR and (b) CR (a)

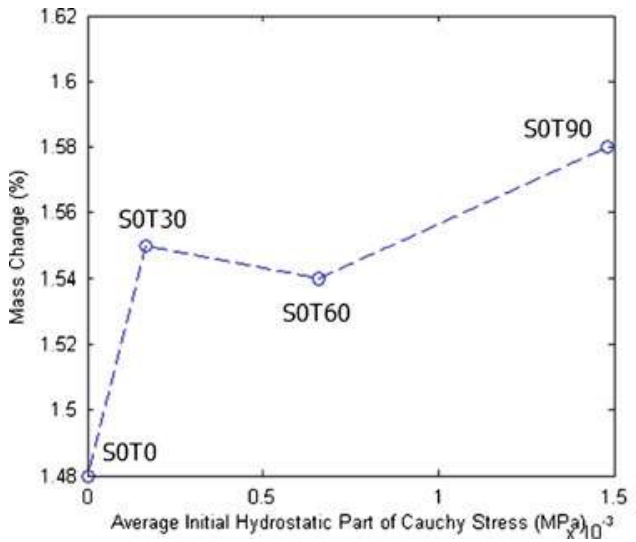

(b)

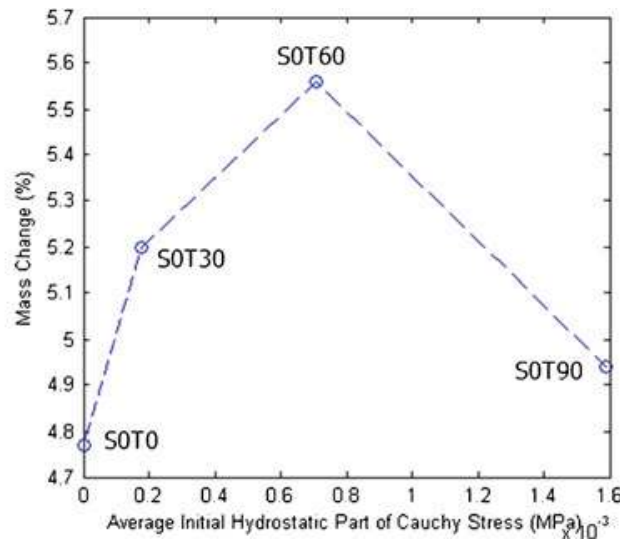




\section{Conclusions}

Novel experimental specimen and device for the investigation of swelling of rubber in the presence of multiaxial large strain has been developed. The device consists of a hollow diabolo rubber specimen attached to specially-designed circular metallic grips and plates such that immersion tests can be conducted while the specimens are simultaneously subjected to various mechanical loading conditions: simple tension, simple torsion and combined tension-torsion.

The levels of swelling experienced by both NBR and $\mathrm{CR}$ are found to be significantly affected by the presence of mechanical loading. More precisely, the effect of tensile strain appeared to be more significant than that of torsion. It was found that the swelling level increases significantly when a tensile strain is introduced. Moreover, slight increase in swelling is observed when a twist is applied. The increase of swelling due to the application of torsion is in contrast to the observation of Loke et al. [19] and Treloar [24]. Our results suggest that the corresponding discrepancy could be attributed to the specimen geometry.

To close the paper, it is to note that although the proposed specimen and device enable the experiment to be carried out successfully, there are some limitations where further modifications and improvements are needed. Works are currently in progress in order to overcome the difficulties.

Acknowledgments The authors greatly appreciate the financial support of the Ministry of Higher Education Malaysia through High Impact Research Grant MOHE-HIR D000008-16001 and by the Institute of Research Management and Consultancy, University of Malaya (UM) under the IPPP Fund Project No.: PV028/2011A.

\section{References}

1. Morton M (1987) Rubber technology. Chapman \& Hall, New York

2. Mars WV, Fatemi A (2002) A literature survey on fatigue analysis approaches for rubber. Int J Fatigue 24(9):949-961

3. Bauman JT (2008) Fatigue, stress and strain of rubber components. Guide for design engineers. Hanser Publications, Ohio

4. Fukumori K, Kurauchi T, Kamigaito O (1990) Swelling behaviour of rubber vulcanizates: 2. Effects of tensile strain on swelling. Polym 31(12):2361-2367

5. Treloar LRG (1950) The swelling of cross-linked amorphous polymers under strain. Trans Faraday Soc 46:783-789

6. Flory PJ (1953) Principles of polymer chemistry. Cornell University Press, New York
7. Treloar LRG (1975) The physics of rubber elasticity. Oxford University Press, USA

8. Andriyana A, Chai AB, Verron E, Johan MR (2012) Interaction between diffusion of palm biodiesel and large strain in rubber: effect on stress-softening during cyclic loading. Mech Res Commun 43:80-86

9. Chai AB, Andriyana A, Verron E, Johan MR, Haseeb ASMA (2011) Development of a compression test device for investigating interaction between diffusion of biodiesel and large deformation in rubber. Polym Test 30:867-875

10. Chester SA, Anand L (2010) A coupled theory of fluid permeation and large deformations for elastomeric materials. J Mech Phys Solids 58(11):1879-1906

11. Jerabek M, Major Z, Lang RW (2010) Uniaxial compression testing of polymeric materials. Polym Test 29(3):302-309

12. Soares JS (2009) Diffusion of a fluid through a spherical elastic solid undergoing large deformations. Int J Eng Sci 47(1):50-63

13. Hong W, Zhao X, Zhou J, Suo Z (2008) A theory of coupled diffusion and large deformation in polymeric gels. J Mech Phys Solids 56(5):1779-1793

14. Baek S, Srinivasa AR (2004) Diffusion of a fluid through an elastic solid undergoing large deformation. Int J Nonlinear Mech 39(2):201-218

15. Azaar K, Rosca ID, Vergnaud JM (2002) Anisotropic swelling of thin epdm rubber discs by absorption of toluene. Polym 43(15):4261-4267

16. Shenoy SL (1998) The effect of uniaxial deformation on swollen gels. Polym Gels Netw 6(6):455-470

17. Mostafa A, Abouel-Kasem A, Bayoumi MR, El-Sebaie MG (2009) Effect of carbon black loading on the swelling and compression set behavior of sbr and nbr rubber compounds. Mater Des 30(5):1561-1568

18. Treloar LRG (1967) The effect of network breakdown and reformation on the swelling of rubbers in compression. Polym $8: 433-442$

19. Loke KM, Dickinson M, Treloar LRG (1972) Swelling of a rubber cylinder in torsion: part 2. Experimental. Polym 13(5):203-207

20. Chai AB, Andriyana A, Verron E, Johan MR (2013) Mechanical characteristics of swollen elastomers under cyclic loading. Mater Des 44:566-572

21. Haseeb ASMA, Jun TS, Fazal MA, Masjuki HH (2011) Degradation of physical properties of different elastomers upon exposure to palm biodiesel. Energy 36(3):1814-1819

22. Zhang H, Cloud A (2007). Research progress in calenderable fluorosilicone with excellent fuel resistance. Arlon Silicone Technologies Division, SAMPE

23. Pekcan Ö, Uğur Ş (2002) Molecular weight effect on polymer dissolution: a steady state fluorescence study. Polym 43(6):19371941

24. Treloar LRG (1972) Swelling of a rubber cylinder in torsion: part 1. Theory. Polym 13(5):195-202

25. Green AE, Adkins JE (1970) Large elastic deformations, vol 2. Clarendon, Oxford

26. Andriyana A, Saintier N, Verron E (2010) Configurational mechanics and critical plane approach: concept and application to fatigue failure analysis of rubberlike materials. Int J Fatigue 32(10):1627-1638

27. Verron E, Andriyana A (2008) Definition of a new predictor for multiaxial fatigue crack nucleation in rubber. J Mech Phys Solids 56(2):417-443

28. Holzapfel GA (2000) Nonlinear solid mechanics: a continuum approach for engineering. Wiley, Chichester 